\title{
Checkerboard superconducting order and antinodal Bogoliubov quasiparticle interference
}

\author{
V. I. Belyavsky, V. V. Kapaev, and Yu. V. Kopaev \\ P. N. Lebedev Physical Institute of Russian Academy of Sciences, Moscow, 119991, Russia
}

\begin{abstract}
Numerical study of momentum-dependent gap function is presented to make clear the origin of superconductivity in copper oxides. We claim that antinodal region with pronounced nesting feature of the Fermi contour gives rise to superconducting pairing with large momentum under screened Coulomb repulsion. Such a pairing results in both spatial checkerboard pattern of the superconducting state below $T_{c}$ and a gapped state of incoherent pairs in a broad temperature range above $T_{c}$. We explain the momentum dependence of the coherent spectral weight detected in angleresolved photoemission spectroscopy and predict antinodal Bogoliubov quasiparticle interference other than observed in the nodal region.

PACS numbers: 78.47.+p, 78.66.-w
\end{abstract}

\section{INTRODUCTION}

Angle resolved photoemission spectroscopy (ARPES) of underdoped cuprates at temperatures exceeding conditional upper boundary $T^{*}$ of the pseudogap (PG) state evidences in favour to large simply connected Fermi contour (FC) typical of the conventional Fermi liquid. However, below $T^{*}$, the $\mathrm{FC}$ is seen as transformed into disconnected arcs disposed in the nodal regions. Cooling from $T^{*}$ down to superconducting (SC) transition temperature $T_{c}$ results in a decrease of arc length down to zero. Thus, the FC degenerates into four points that give rise to the nodes of $d$ - wave SC order parameter arising below $T_{c}$. It seems fairly natural to conclude that, within the framework of $d$ - wave pairing concept, the SC order parameter has its maximal value exactly in the antinodal directions. $\underline{\underline{1}}$

For this reason, it might seem quite probable that low-temperature properties of $d$ - wave superconductor should be determined by low-energy quasiparticle excitations only in the nodal region of the momentum space that is in vicinities of the SC gap nodes on the diagonals of the Brillouin zone. Taking into account that Bogoliubov quasipatricle interference (QPI), observed in the nodal region, disappears near the end points of the FC $\operatorname{arcs}, \stackrel{2}{,}$ one might lead to a conclusion that only the nodal region gives rise to superconductivity whereas the gap observed in the antinodal region should be attributed to an incoherent PG state $\stackrel{2}{2}$ However, a coherence in the antinodal region becomes apparent both in the ARPES study ${ }^{\underline{3}}$ and also in the Andreev - St James experiments $\underline{\underline{4}}$ Therefore, in spite of the fact that high-energy QPI is not detected for the present, one can believe that the antinodal region should contribute a coherent state as well.

We have $\operatorname{argued}^{5}$ that both PG and SC states arise exactly in the antinodal region with pronounced nesting of the FC as spatially inhomogeneous incoherent and coherent states of pairs with large momentum, respectively. The nodal region gives rise to conventional SC pairing with zero momentum which, together with the pairing with large momentum ( $\boldsymbol{K}$-pairing) in the antin- odal region, forms a biordered SC state in the whole of the Brillouin zone.

Kinematic constraint, inherent in $\boldsymbol{K}$-pairing in the antinodal region, can result in oscillating real-space pairing interaction. Indeed, momenta of both particles composing SC pair with nonzero total momentum $\boldsymbol{K}$ should be either inside or outside of the FC. For this reason, a set of one-particle states turns out to be kinematically excluded because of the fact that such states cannot contribute into the states of $\boldsymbol{K}$-pairs. It means that any scattering between such excluded states should be forbidden when one defines the interaction leading to a rise of a bound state of $\boldsymbol{K}$-pair. An exclusion of a set of the Fourier components from the screened Coulomb interaction results in the fact that corresponding real-space $\boldsymbol{K}$-pairing interaction exhibits an oscillation outside of small-distance repulsive core as shown schematically in Fig. 1. It should be noted that there is an analogy between this oscillation and well-known Friedel oscillation that arises owing to Kohn singularity of screening enhanced by nesting of the FC.

Besides the fact that two-particle problem with oscillating potential leads to a bound state of the relative motion of $\boldsymbol{K}$-pair, $\frac{6}{\underline{6}}$ it can also produce a quasistationary state $(\mathrm{QSS})^{7}$ similar to the Gamov's state of alpha-radioactive nucleus $\stackrel{8}{\mathrm{~S} C}$ gap function $\Delta_{s c}(\boldsymbol{k})$, depending on relative-motion momentum $\boldsymbol{k}$ of $\boldsymbol{K}$-pair, as a solution to the mean-field self-consistency equation, arises as a result of the instability of the ground state of the normal Fermi liquid with respect to a rise of $\boldsymbol{K}$-pairs in the bound state. This function can be expressed in terms of Gorkov's anomalous averages describing SC condensate of $\boldsymbol{K}$-pairs. Due to a phase coherence of the SC ground state, these averages become nonzero below $T_{c}$. QSS with positive energy, following from the two-particle problem, can be considered as an evidence in favor to one more instability of the Fermi liquid. We associate such an instability with incoherent $\boldsymbol{K}$-pairs existing above $T_{c}$. It means that Gorkov's anomalous averages and corresponding gap function $\Delta_{p g}(\boldsymbol{k})$ vanish under averaging over phases of $\boldsymbol{K}$-pairs but mean square gap function 


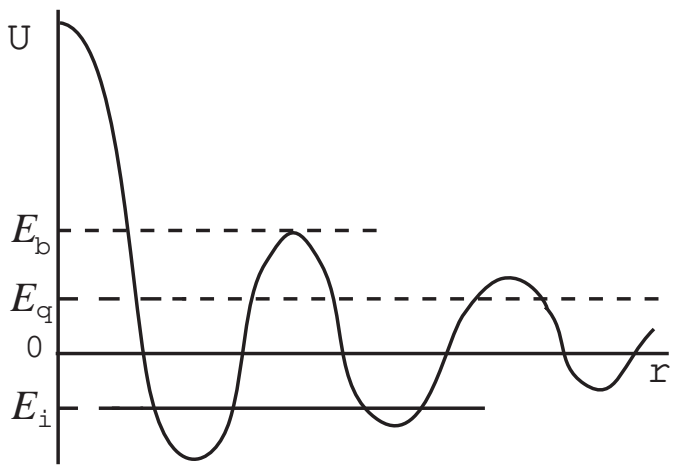

FIG. 1: Real-space pairing potential $U(r)$ (schematically). Energies $E_{i}$ and $E_{q}$ correspond to bound and quasi-stationary states, respectively. Barrier height $E_{b}$ corresponds to a break of the pair without tunnelling through the barrier.

remains nonzero up to $T^{*}$ according to the hypothesis advanced by Emery and Kivelson. $\underline{9}$

Thus, one can conclude that, in the temperature range from $T^{*}$ down to $T_{c}, \boldsymbol{K}$-pairs form incoherent PG state as off-diagonal short-range order (ODSRO). Off-diagonal long-range order (ODLRO) arises as SC condensate of coherent $\boldsymbol{K}$-pairs below $T_{c}$. Both ODLRO and ODSRO states can be described in terms of Gorkov's Green functions. ${ }^{10} \mathrm{~A}$ phenomenological BCS-like form of the coherent contribution to the normal Gorkov's function can be written as

$$
G(\omega ; \boldsymbol{k})=z(\boldsymbol{k})\left[\frac{u_{+}^{2}(\boldsymbol{k})}{\omega-E(\boldsymbol{k})+i \Gamma}+\frac{u_{-}^{2}(\boldsymbol{k})}{\omega+E(\boldsymbol{k})-i \Gamma}\right],
$$

where $E(\boldsymbol{k})$ and $2 u_{ \pm}^{2}(\boldsymbol{k})=1 \pm \xi(\boldsymbol{k}) / E(\boldsymbol{k})$ are quasiparticle energy and coherence factors, respectively,

$$
2 \xi(\boldsymbol{k})=\varepsilon(\boldsymbol{K} / 2+\boldsymbol{k})+\varepsilon(\boldsymbol{K} / 2-\boldsymbol{k})
$$

is the kinetic energy of the $\boldsymbol{K}$ - pair of particles with momenta $\boldsymbol{K} / 2 \pm \boldsymbol{k}, \varepsilon(\boldsymbol{k})$ is electron dispersion with respect to chemical potential $\mu$ and $z(\boldsymbol{k})$ is quasiparticle weight. Two terms in $G(\omega ; \boldsymbol{k})$ can be referred to $\boldsymbol{K}$-pairs above and below the FC, respectively. Diagonal Green function $G(\omega ; \boldsymbol{k})$ describes ODSRO state corresponding to the existence of non-coherent QSS of $\boldsymbol{K}$ - pairs above $T_{c}$. Transition from the bound paired state into long-living QSS corresponds to small but finite decay $\Gamma=\Gamma(\omega ; \boldsymbol{k})$ whereas transitions into stationary states above barrier energy $E_{b}$ (Fig. 1) should be associated with an infinitesimal decay, $\gamma \rightarrow+0$, leading to conventional Fermi-liquid behavior of $G(\omega ; \boldsymbol{k})$ above $T^{*}$. Thus, a rise of QSS results in a non-Fermi-liquid behavior of $G(\omega ; \boldsymbol{k}))$ that can be related to the $\mathrm{PG}$ state.

The SC state below $T_{c}$ should be described by both normal and anomalous Gorkov's functions. Taking into account the fact that PG function $\Delta_{p g}(\boldsymbol{k})$, averaged over random phases, vanishes whereas $\Delta_{s c}(\boldsymbol{k}) \neq 0$ below $T_{c}$, one can introduce anomalous Gorkov's function $F^{+}(\omega ; \boldsymbol{k})$ in a way we use to obtain $G(\omega ; \boldsymbol{k})$ :

$$
F^{+}(\omega ; \boldsymbol{k})=-z(\boldsymbol{k}) \frac{\Delta_{s c}^{*}(\boldsymbol{k})}{(\omega-E(\boldsymbol{k})+i \Gamma)(\omega+E(\boldsymbol{k})-i \Gamma)} .
$$

Such an approach directly leads to uniform description of both SC and PG states in underdoped cuprates. One can see that repulsive Coulomb pairing in the antinodal region necessarily results in rather complicated momentum dependence of the SC gap and PG functions, $\Delta_{s c}(\boldsymbol{k})$ and $\Delta_{p g}(\boldsymbol{k})$, with energy scale $\varepsilon_{0} \sim 1 \mathrm{eV}$ of their domains of definition ${ }^{11}$ in contrast to considerably less scale of about Debye energy $\varepsilon_{D}$ that arises in the case of phonon-mediated SC pairing. It is very likely that the high energy problem, $\frac{12}{2}$ arising, in particular, in the optical conductivity of the cuprates, 13 might be associated with high energy scale of the antinodal $\boldsymbol{K}$-pairing. We believe that $\boldsymbol{K}$-pairs are the main players in the hightemperature superconductivity of the cuprates.

Recently, Tsvelik and Chubukov ${ }^{14}$ considered SC pairing on mutually orthogonal pairs of perfectly nested segments of the FC in semiphenomenological way. They presuppose that one-dimensional SC order arises only on these segments coupled with a momentum-space Josephson links to give rise to two-dimensional superconductivity. Actually, it is implicitly supposed that such SC state can arise owing to SC pairing with nonzero momentum. Also, it is supposed that SC order with the same momentum is induced on the rest unnested part of the FC by the order on the nested segments due to a proximity effect in the momentum space ${ }^{6}$ so that this induced order cannot penetrate deep into the nodal region. It should be emphasize that such a mode ${ }^{14}$ differs essentially from the biordered SC state. $\frac{5}{}$

In this paper, we study the mean-field $\boldsymbol{K}$-pairing problem numerically to fall outside the weak coupling limits employed in our previous approach to the $\boldsymbol{K}$-pairing problem 5,6.7 We show that the $\mathrm{SC}$ gap function with a nontrivial nodal line corresponds to a checkerboard pair density wave (PDW) SC state and results in fairly natural explanation of the angle dependence of a partial suppression of the coherent spectral weight in the antinodal region observed by Kondo et al $\stackrel{3}{3}^{-}$We believe that QPI, other than observed in the nodal region, ${ }^{2}$ could be detected in the antinodal one as well. We also show that $\boldsymbol{K}$ pairing can originate spatial checkerboard pattern without any driving insulating order in contrast to a scenario of a rise of PDW coexisting with a charge density wave (CDW) 15

\section{II. $K$ - PAIRING PROBLEM}

In the case of $\boldsymbol{K}$-pairing, the gap function is defined as

$$
\Delta(\boldsymbol{k})=\sum_{\boldsymbol{k}^{\prime}} U\left(\boldsymbol{k}, \boldsymbol{k}^{\prime}\right)\left\langle\hat{c}_{\boldsymbol{K} / 2-\boldsymbol{k}^{\prime} \downarrow} \hat{c}_{\boldsymbol{K} / 2+\boldsymbol{k}^{\prime} \uparrow}\right\rangle
$$


where $U\left(\boldsymbol{k}, \boldsymbol{k}^{\prime}\right)$ is screened Coulomb interaction matrix element, operator $\hat{c}_{\boldsymbol{k} \sigma}$ annihilates electron with momentum $\boldsymbol{k}$ and spin polarization $\sigma$. Anomalous average in Eq. (2), describing SC condensate of $\boldsymbol{K}$ - pairs, becomes nonzero below $T_{c}$. The gap function should be a nontrivial solution to the self-consistency equation,

$$
\Delta(\boldsymbol{k})=-\frac{1}{2} \sum_{\boldsymbol{k}^{\prime}} \frac{U\left(\boldsymbol{k}, \boldsymbol{k}^{\prime}\right) \Delta\left(\boldsymbol{k}^{\prime}\right)}{\sqrt{\xi^{2}\left(\boldsymbol{k}^{\prime}\right)+\Delta^{2}\left(\boldsymbol{k}^{\prime}\right)}}\left[1-n\left(\boldsymbol{k}^{\prime}\right)\right] .
$$

Here, $n(\boldsymbol{k})=\left(e^{E(\boldsymbol{k}) / T}+1\right)^{-1}$ is a quasiparticle occupation number and quasiparticle energy has the form

$$
\begin{gathered}
E(\boldsymbol{k})=\eta(\boldsymbol{k}) \pm \sqrt{\xi^{2}(\boldsymbol{k})+\Delta^{2}(\boldsymbol{k})} \\
2 \eta(\boldsymbol{k})=\varepsilon(\boldsymbol{K} / 2+\boldsymbol{k})-\varepsilon(\boldsymbol{K} / 2-\boldsymbol{k}) .
\end{gathered}
$$

It should be noted that, since $\varepsilon(-\boldsymbol{k})=\varepsilon(\boldsymbol{k})$ owing to the time-reversal symmetry of the dispersion relation, quasiparticle spectrum (4) turns out to be gapped on the whole of the FC in the case of pairing with zero total momentum. In the case of $\boldsymbol{K}$-pairing, it can be gapped only on those parts of the FC where $|\eta(\boldsymbol{k})|$ proves to be small enough. In addition, the quasiparticle spectrum becomes asymmetrical with respect to $\mu$.

Summation in Eqs. (2) and (3) should be performed over all momenta of the relative motion which can form pairs with given total momentum $\boldsymbol{K}$. One can see that these momenta belong to a $\boldsymbol{K}$ - dependent domain of the momentum space (domain of kinematic constraint) because of the fact that the momenta of both particles composing SC pair with given total momentum should be situated either inside or outside of the FC. This means that some part of the momentum space turns out to be excluded from the sums in Eqs. (23).

Since the kinetic energies of both particles composing SC pair with $\boldsymbol{K}=0$ can be equal to $\mu$, the low-energy limit in the sum (3) corresponds to $\xi=0$ whereas the upper limit is formally restricted by a half-width of the conduction band of the order of $\mu$. However, in the BardeenCooper-Schrieffer (BCS) theory $\underset{16}{r}$ such upper limit (Debye phonon energy $\varepsilon_{D}$ ) appears as an energy scale of a layer enveloping the FC where electron-electron scattering results in an effective attraction between electrons. As a result, pairing interaction energy $U\left(\boldsymbol{k}, \boldsymbol{k}^{\prime}\right)$ in Eq. (3) can be qualitatively associated with an effective coupling constant $V^{*}$ that can be estimated as 17

$$
V^{*}=V-\frac{U}{1+U g \ln \left(\mu / \varepsilon_{D}\right)},
$$

where $g$ is density of states per spin, $V$ is a pairing constant due to electron-phonon interaction defined inside the layer, $U$ is average Coulomb energy. Thus, in the effective pairing constant, Coulomb repulsion appears with a logarithmic weakening. In the case when $V^{*} g \ll 1$, the mean-field approach results in a conventional BCS energy gap,

$$
\Delta=2 \varepsilon_{D} \exp \left(-1 / V^{*} g\right),
$$

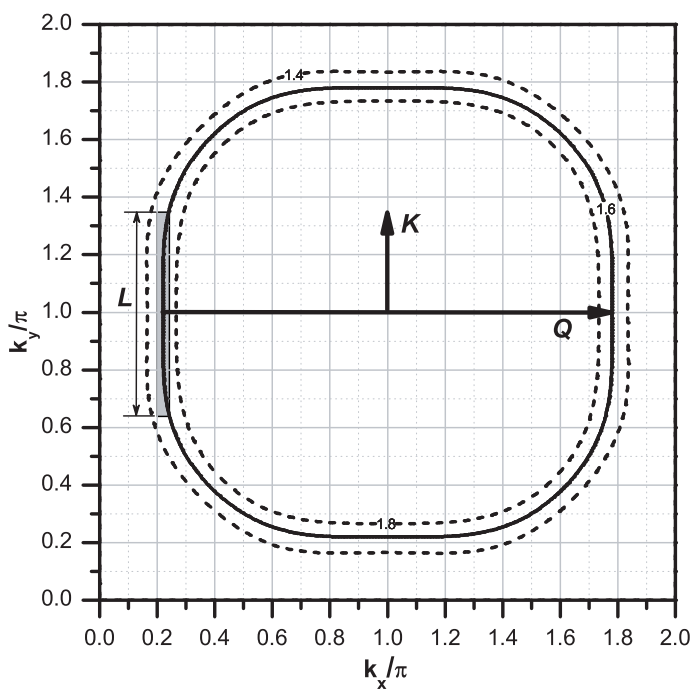

FIG. 2: Near nested FC (solid line) corresponding to electron dispersion Eq. (9) typical of the cuprates. Dashed lines represent isolines close to the $\mathrm{FC}$, numbers near the isolines are electron energies according to Eq. (9) with $t=0.5, t^{\prime}=-0.15$, $t^{\prime \prime}=0.07 \mathrm{eV}$. Here, $\boldsymbol{K}$ is total momentum of $\boldsymbol{K}$-pair, $\boldsymbol{Q}$ is nesting momentum. Length $L$ of near rectilinear segment of the FC is shown at given mean square energy deviation $\delta$ (the width of the shadowed strip) of the FC from the rectilinearity.

that appears in consequence of the logarithmic singularity of the right-hand side of Eq. (3). This singularity is primarily formed in an energy range near the low-energy limit, therefore, extension of this range might lead to a progressive accumulation of the singularity along with the formation of a non-singular (regular) contribution into Eq. (3). One can treat the preexponential in Eq. (7) as a characteristic energy scale beyond which the nontrivial solution to the self-consistency equation becomes weakly sensitive to the upper limit.

All these speculations can be referred to the $\boldsymbol{K}$ - pairing problem. However, in such a case, the logarithmic singularity becomes apparent if and only if kinetic energy of $\boldsymbol{K}$ - pair vanishes not at isolated points, as it were most likely in the case of arbitrary FC, but on finite pieces of the FC on which mirror nesting condition,

$$
\varepsilon(\boldsymbol{K} / 2+\boldsymbol{k})-\varepsilon(\boldsymbol{K} / 2-\boldsymbol{k})=0,
$$

should be fulfilled at given $\boldsymbol{K}$. One can see that, for a suitable $\boldsymbol{K}$, this condition can be fulfilled in the case of rectilinear parallel segments on the opposite sides of the FC. It is obvious that $\boldsymbol{K}$ should be directed along these segments.

FC, typical of the cuprates, and isolines close to it can be described satisfactorily by electron dispersion

$$
\begin{aligned}
\varepsilon\left(k_{x}, k_{y}\right) & =t_{0}-2 t\left(\cos k_{x}+\cos k_{y}\right)-4 t^{\prime} \cos k_{x} \cos k_{y}- \\
& -2 t^{\prime \prime}\left(\cos 2 k_{x}+\cos 2 k_{y}\right)
\end{aligned}
$$

with fitting parameters $t_{0}=2 \mathrm{eV} t=0.5 \mathrm{eV}, t^{\prime} / t=$ $-0.3, t^{\prime \prime}=0.14$. Here, $k_{x}$ and $k_{y}$ (in units of $\pi / a$; $a$ is 
interatomic distance) are momentum components corresponding to the antinodal directions.

One can choose one of the coordinate axes $\left(k_{y}\right)$ along $\boldsymbol{K}$ directed parallel to antinodal near rectilinear segments of the FC as shown in Fig. 2. Then, with preassigned accuracy $\delta, \xi(\boldsymbol{k}) \leq \delta$ if $k_{y}$ corresponds to near rectilinear segment of the FC. Therefore, the singular contribution into (3) turns out to be proportional to the length $L$ of such a segment. Summation over the other component $\left(k_{x}\right)$ leads to an accumulation of the singularity, however, in contrast to the case $\boldsymbol{K}=0$, a gradual deviation from the FC results in a progressive increase of the difference between the kinetic energies of the particles composing $\boldsymbol{K}$ - pair. This leads to increasing deviation from mirror nesting condition (8), so that, finally, the accumulation turns out to be completed when $k_{x}$ attains a value corresponding to energy scale $\varepsilon_{0}$ much lesser than $\mu$. It should be noted that, in the case of $\boldsymbol{K}$ - pairing, $\varepsilon_{0}$ appears as generic energy scale originating from mirror nesting feature of electron dispersion. This scale should be related to a preexponential of the gap function in the case of small effective coupling constant. Thus, one can conclude that the nontrivial solution to the self-consistency equation should be weakly sensitive to the part of the momentum space corresponding to $\varepsilon>\varepsilon_{0}$.

Since equality (8) is fulfilled only approximately, one can choose length $L$ in order that mean square deviation of the FC from the rectilinearity were less than a preassigned value corresponding to energy scale $\delta$. Strictly speaking, nonzero $\delta$ eliminates the singularity because of a rise of a lower limit cutoff in the sum Eq. (3). Similar cutoff appears in the Fulde - Ferrell - Larkin - Ovchinnikov (FFLO) problem of SC pairing with small total pair momentum. $\stackrel{18,19}{ }$ Therefore, nontrivial solution to equation (3) can exist if the effective coupling constant exceeds certain $\delta$ - dependent value. Magnitude $\Delta^{\prime}$ of the gap function can be roughly estimated as

$$
\Delta^{\prime}=\sqrt{\Delta(\Delta-2 \delta)},
$$

where $\Delta$ is the magnitude corresponding to perfect mirror nesting that is to exactly rectilinear segment of the FC with length $L$. Positive function $\Delta^{\prime}(\delta)$ has a maximum at certain $\delta \equiv \delta_{m}<\Delta / 2$. Indeed, $\Delta^{\prime} \rightarrow 0$ if $\delta \rightarrow 0$ (then, generally speaking, $L \rightarrow 0$, so that there is no singularity in the self-consistency equation: $\Delta \rightarrow 0$ ). At $2 \delta>\Delta$, the magnitude of the gap function vanishes, therefore, a maximum value of $\Delta^{\prime}$ exists at $0<\delta_{m}<\Delta / 2$. A choice of length $L$ of near rectilinear segment of the FC at given $\delta$ predetermines total pair momentum $\boldsymbol{K}$. It is clear that, because of kinematic constraint, the absolute value of $\boldsymbol{K}$ coincides with $L / 2$, as one can see from Fig. 2. Since maximum value of $\Delta^{\prime}$ corresponds to $\delta_{m}$, the absolute value of the momentum of $\boldsymbol{K}$ - pairs in the SC condensate should be taken as $K=L\left(\delta_{m}\right) / 2$. Variation of the FC with doping $x$ in hole doped cuprates 20 results in a conclusion that $K$ should be dependent on $x$. Note that there is no contradiction between such a dependence, following from dispersion (9), and doping

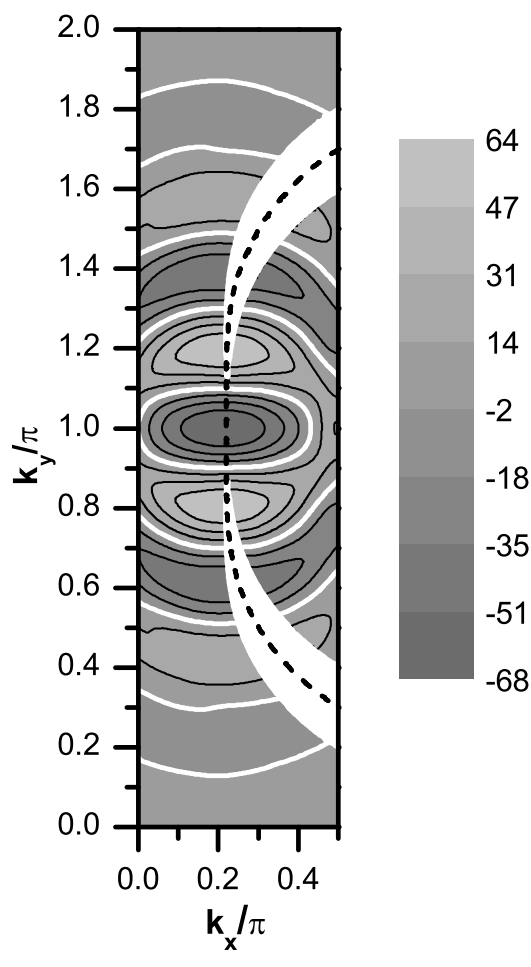

FIG. 3: Calculated momentum dependence of the antinodal gap function (gradation of grey, in $m e V$ ) shown in a region of the Brillouin zone that primarily forms the singularity of the self-consistency equation. White curves represent the nodal line structure of the gap function, dashed line is the FC corresponding to that shown in Fig. 2. White regions, in which the nontrivial solution is absent due to the kinematic constraint, appear under shifting of the $\mathrm{FC}$ at $\pm \boldsymbol{K} / 2$ along $k_{y}$ (inside these regions, there are no one-particle states that could form a pair with total momentum $K=0.2 \pi / a$ ) corresponding to a maximum magnitude of the gap function.

dependence of spatial periodicity of checkerboard PDW seen in tunnel data. 21

Comparatively small vicinity with energy scale $\varepsilon_{0}$ of the strip with length $L\left(\delta_{m}\right) / 2$ and width corresponding to energy scale $\delta_{m}$ can be considered as the region of the momentum space that primarily forms the singularity of the self-consistency equation. Following Ref. [10], one can renormalize the kernel of this equation and reduce Eq. (3) to a sum over momenta belonging to such a vicinity only. Renormalized kernel, defined in this vicinity, can be written as 22

$$
W\left(\boldsymbol{k}, \boldsymbol{k}^{\prime}\right)=\sum_{n} \frac{\phi_{n}(\boldsymbol{k}) \phi_{n}^{*}\left(\boldsymbol{k}^{\prime}\right)}{\lambda_{n}+g \ln \left(\mu / \varepsilon_{0}\right)},
$$

and can be treated as a pairing pseudopotential corresponding to oscillating real-space pairing interaction. Here, $\phi_{n}(\boldsymbol{k})$ and $\lambda_{n}$ are eigenfunctions and eigenvalues of kernel $U\left(\boldsymbol{k}, \boldsymbol{k}^{\prime}\right)$, respectively $\underline{\underline{6}}$ We believe that the vicinities with energy scale $\varepsilon_{0}$ of the antinodal near rectilinear segments of the FC include electron states that mainly contribute to scattering resulting in $\boldsymbol{K}$-pairing. 
Since characteristic sizes of the vicinity are much less than characteristic Fermi momentum, region of attraction in the real space proves to be more deep and extended with respect to that due to Friedel oscillation. Such oscillating interaction can provide both bound state and QSS of the relative motion of $\boldsymbol{K}$ - pair. In the mean-field approach, the bound state appears in temperature range $0 \leq T<T_{c}$ as nonzero anomalous averages, $\left\langle\hat{c}_{\boldsymbol{K} / 2-\boldsymbol{k} \downarrow} \hat{c}_{\boldsymbol{K} / 2+\boldsymbol{k} \uparrow}\right\rangle \neq 0$, that determine gap function Eq. (2). It should be noted that, in the case of small $\boldsymbol{K}$ (for example, in the FFLO state), real-space oscillation of the pairing interaction becomes weak enough because of considerable extension of the corresponding vicinity forming the singular contribution into the selfconsistency equation.

To study $\boldsymbol{K}$-pairing problem numerically, we use a step-wise approximation of the pairing interaction 11 assuming that pseudopotential (11) has a constant value of about $10 \mathrm{eV}$ inside a vicinity of near rectilinear segments of the FC. Energy scale of such a vicinity is determined from the above mentioned condition that calculated gap function magnitude should become actually independent of this scale beginning with certain $\varepsilon_{0}$.

Numerical study of Eq. (3) at $T=0$ reveals highly complicated momentum dependence of gap function $\Delta(\boldsymbol{k})$, shown in Fig. 3, with a few closed nodal lines crossing the FC. Topological feature of the gap function, shown only inside the part of the Brillouin zone that primarily contributes into the singularity of the selfconsistency equation, turns out to be weakly dependent on small variation of the parameters of electron dispersion and magnitude of pairing interaction. According to rough estimation following from (10), maximum value of $K$ - dependent magnitude of the gap function can be associated with $K$ close to $0.2 \pi / a$. Domain of definition of the pairing pseudopotential includes all energies $\varepsilon<\varepsilon_{0}$, where $\varepsilon_{0}$ is relative to a distance between the $\mathrm{FC}$ and the boundary of this domain. As follows from numerical solution to Eq. (3), a gradual decrease of the momentum corresponding to upper limit $k_{r}$ in the sum over $k_{x}$ in the self-consistency equation with renormalized kernel, at first, does not affect the magnitude of the gap function. Then, beginning with certain value of $k_{r}$, that can be associated with a boundary of the domain of definition of $W\left(\boldsymbol{k}, \boldsymbol{k}^{\prime}\right)$, the magnitude tends to zero with a decrease of $k_{r}$. This gives a possibility to determine energy scale $\varepsilon_{0} \approx 0.3 \mathrm{eV}$ of this domain forming the singularity of the self-consistency equation.

\section{CHECKERBOARD ODLRO}

Visualization of a checkerboard $\mathrm{PDW}^{21}$ can be considered as an indirect evidence in favour of the fact that such a state originates from nesting feature of the FC typical of the cuprates. It should be noted that near rectilinear segments on the opposite sides of such FC ensure not only mirror nesting condition (8) but also nesting condition

$$
\varepsilon(\boldsymbol{k}+\boldsymbol{Q})+\varepsilon(\boldsymbol{k})=0
$$

at certain nesting momentum $\boldsymbol{Q}$ which, in general, is incommensurate with total momentum $\boldsymbol{K}$ of SC pair as shown in Fig. 2. Under condition (12), the logarithmic singularity can arise in an insulating pairing channel that gives rise, for example, to CDW. Such an insulating pairing can compete or coexist with $\boldsymbol{K}$ - pairing in a way considered a long time ago in the case of the coexistence of conventional $(K=0)$ SC state and CDW $\underline{\underline{24}}$

One can compare efficiencies of both channels with the help of a crude estimation of lengths $L_{K}$ and $L_{Q}$ of near rectilinear segments forming singularities in the SC and insulating channels, respectively. These lengths, at given $\delta$, the same in both channels, can be found from inequalities

$$
\begin{array}{r}
|\varepsilon(\boldsymbol{K} / 2+\boldsymbol{k})-\varepsilon(\boldsymbol{K} / 2-\boldsymbol{k})| \leq \delta, \\
|\varepsilon(\boldsymbol{k}+\boldsymbol{Q})+\varepsilon(\boldsymbol{k})| \leq \delta,
\end{array}
$$

selecting the regions in the momentum space in which mirror nesting or nesting condition, respectively, is satisfied with preassigned accuracy. If boundaries of these regions intersect the FC, lengths $L_{K}$ and $L_{Q}$ should be defined as distances between the corresponding intersection points. Both pair momentum $\boldsymbol{K}$ and nesting momentum $\boldsymbol{Q}$ should be selected in a way to ensure maximum values of corresponding lengths $L_{K}$ and $L_{Q}$, respectively. Momenta $\boldsymbol{K}$ and $\boldsymbol{Q}$ depend on a form of the FC varying with doping. Therefore, interrelation between $L_{K}$ and $L_{Q}$ varies with doping as well. Calculated variations of $L_{K}$ and $L_{Q}$ with doping are shown schematically in Fig. 4. A comparison of $L$ and $L_{Q}$ shows that, in the case of electron dispersion Eq. (9), nesting dominates mirror nesting in electron doped compounds. On the contrary, the opposite case of hole doping gives an opportunity of a rise of such a range of $x$ where mirror nesting dominates nesting.

In this range, $\mathrm{SC}$ order arises due to $\boldsymbol{K}$ - pairing whereas insulating order (CDW with $Q=2 K$ ) with low spectral weight can exist as induced by the corresponding PDW and, therefore, turns out to be hardly detected. Conversely, dominating insulating order in the form of CDW can induce the PDW $\underline{23}$ as a modulated superfluid density which, due to low spectral weight, seems to be undetectable. Thus, one can conclude that, if mirror nesting dominates nesting, SC state in the form of PDW of $\boldsymbol{K}$ - pairs can exist without any driving insulating order.

It should be emphasized that nesting feature of the FC can lead to a giant enhancement of the singularity in both pairing channels. ${ }^{24.25}$ In the insulating channel, nesting of the FC transforms weak Kohn anomaly into the CDW. In the SC channel, mirror nesting gives rise to $\boldsymbol{K}$-pairing which, owing to kinematic constraint, ensures extended and deep oscillation of real-space screened Coulomb pairing interaction and, consequently, results in $T_{c}$ considerably greater than following from Kohn-Luttinger SC pairing 26 with $\boldsymbol{K}=0$ and angular momentum $l \neq 0$. 


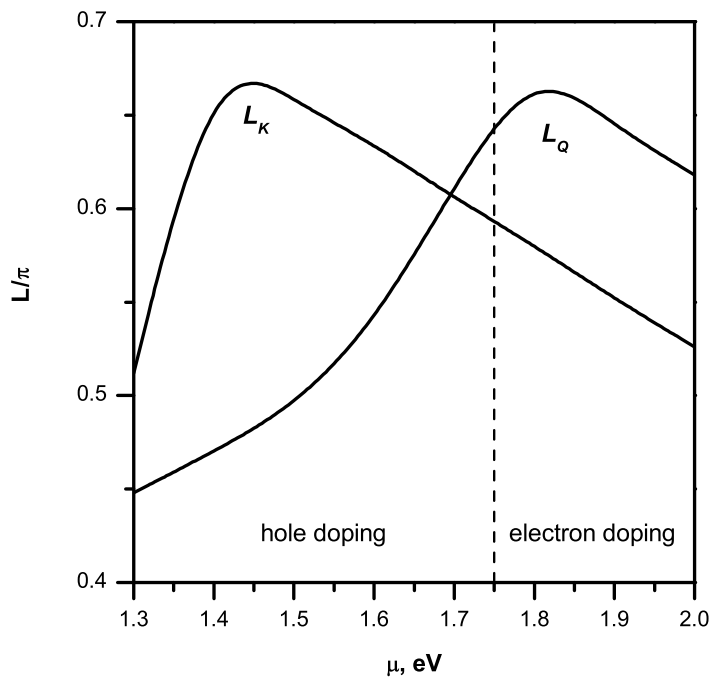

FIG. 4: Competition between nesting and mirror nesting: doping dependence of lengths $L_{Q}$ and $L$ of the optimal near rectilinear segments of the $\mathrm{FC}$ under nesting and mirror nesting conditions, respectively.

Thus, $\boldsymbol{K}$-pairing leads to an independent order so that, in such a case, there is no need to take into account a coexistence of $\mathrm{SC}$ and insulating ordered states $\frac{15}{5}$ to invoke spatially inhomogeneous SC state in the form of PDW.

One can define anomalous averages $\left\langle\hat{\psi}_{\downarrow}\left(\boldsymbol{r}^{\prime}\right) \hat{\psi}_{\uparrow}(\boldsymbol{r})\right\rangle$ in the real space that corresponds to the momentum-space anomalous averages, $\left\langle\hat{c}_{\boldsymbol{K} / 2-\boldsymbol{k} \downarrow} \hat{c}_{\boldsymbol{K} / 2+\boldsymbol{k} \uparrow}\right\rangle$, arising due to $\boldsymbol{K}$-pairing. Here, fermion field operator $\hat{\psi}_{\sigma}(\boldsymbol{r})$ annihilates electron with spin polarization $\sigma$ and radius vector $\boldsymbol{r}$. Nonzero anomalous averages can be considered as an order parameter corresponding to $\boldsymbol{K}$-pairing. In the case of the two-dimensional $C_{4}$ orbital symmetry, there are four crystal equivalent pair momenta $\boldsymbol{K}_{j}, j=1,2,3,4$. Therefore, real-space representation of the order parameter should be written as a superposition

$$
\left\langle\hat{\psi}_{\downarrow}\left(\boldsymbol{r}^{\prime}\right) \hat{\psi}_{\uparrow}(\boldsymbol{r})\right\rangle=\sum_{j=1}^{4} \gamma_{j} \varphi_{j}(\boldsymbol{\rho}) \cdot \exp \left(i \boldsymbol{K}_{j} \boldsymbol{R}\right),
$$

where $\boldsymbol{R}=\left(\boldsymbol{r}+\boldsymbol{r}^{\prime}\right) / 2, \boldsymbol{\rho}=\boldsymbol{r}-\boldsymbol{r}^{\prime}$ are center-of-mass and relative motion radius vectors of $\boldsymbol{K}$-pair,

$$
\varphi_{j}(\boldsymbol{\rho})=\frac{1}{N} \sum_{\boldsymbol{k}}\left\langle\hat{c}_{\boldsymbol{K}_{j} / 2-\boldsymbol{k} \downarrow} \hat{c}_{\boldsymbol{K}_{j} / 2+\boldsymbol{k} \uparrow}\right\rangle \exp (i \boldsymbol{k} \boldsymbol{\rho})
$$

can be considered as a real-space wave function of the relative motion of $\boldsymbol{K}$-pair. Here, $N$ is a number of unit cells of the two-dimensional system, summation over $\boldsymbol{k}$ should be performed inside the domain of kinematic constraint corresponding to each momentum $\boldsymbol{K}_{j}$. Coefficients $\gamma_{j}$, corresponding to SC state, should be determined by one of the irreducible representations of the symmetry group $C_{4}$. A choice of the irreducible representation establishes the orbital symmetry of the order parameter. Since $\gamma_{1}=-\gamma_{2}=\gamma_{3}=-\gamma_{4}$ in the case of $d$-wave orbital symmetry, a checkerboard spatial pattern of the order parameter follows from Eq. (14) immediately. One can see that $d$-wave order parameter (14) corresponds to a current-less SC state, therefore, in this respect, it is similar to Larkin - Ovchinnikov immobile wave solution, $\frac{19}{1}$ in contrast to Fulde - Ferrell running wave, 18 of the FFLO problem.

Nonzero anomalous average $\left\langle\hat{c}_{\boldsymbol{K}_{j} / 2-\boldsymbol{k} \downarrow} \hat{c}_{\boldsymbol{K}_{j} / 2+\boldsymbol{k} \uparrow}\right\rangle$ appears as a result of averaging of the product of two annihilation fermion operators over the canonical ensemble in which total particle number $N$ fluctuate with respect to certain mean value $\bar{N}: \underline{16}$ In such an ensemble, all of the states with different $N$ close to $\bar{N}$ should be coherent so that pair correlation function Eq. (14) describes ODLRO of $\boldsymbol{K}$ - pairs in the SC condensate. Above $T_{c}$, phase coherence of the ground state turns out to be lost due to the fact that $\boldsymbol{K}$-pairs in the states with different $N$ have got random center-of-mass phases. Owing to above-mentioned instability of the ground state with respect to a rise of QSS of $\boldsymbol{K}$-pair, relative motion phase of the wave function of such a pair, included into coefficients $\gamma_{j}$, can remain locked up to temperatures far above $T_{c}$.

One can think that a loss of relative-motion phase coherence with heating might go through two steps. At first, $d$-wave current-less superposition (14) can be decomposed into two orthogonal dimer superpositions with $\gamma_{1}= \pm \gamma_{3}, \gamma_{2}=\gamma_{4}=0$ and $\gamma_{2}= \pm \gamma_{4}, \gamma_{1}=\gamma_{3}=0$. After that, at greater temperature, dimer state can be disintegrated into free $\boldsymbol{K}$-pairs which survives up to their break at a temperature that can be associated with the upper boundary of the PG state. Temperature range, corresponding to lost center-of-mass phase coherence but survived relative-motion phase coherence, can be referred to the region of the PG state in which off-condensate SC pairs can appear as spatially inhomogeneous ODSRO. We believe that spatial pattern, like that observed by Kohsaka et al., 2 is described by current-less superpositions Eq. (14) in which coefficients $\gamma_{j}$ correspond to random dimer configurations.

Recently, Berg et al ${ }^{27}$ have considered dimer-like ("striped") ODLRO in the framework of the concept of SC pairing with large momentum. Note that, as follows from numerical study of Hubbard model on $4 \times 4$ square lattice, ${ }^{28} d$-wave checkerboard order as the ground state seems to be favorable with respect to dimer-like one.

Momentum dependence of ODLRO parameter $\Delta_{s c}(\boldsymbol{k})$ determines the angle dependence of the spectral weight, $W_{C P}(\phi)$, of the SC coherent peak appearing in the ARPES spectra below $T_{c}$. In the case of $d$-wave superconductor, it is tacitly assumed that SC order parameter, taken on the FC, is proportional to $\cos 2 \phi$, where Fermi angle $\phi$ is polar angle in the momentum space counted from the antinodal direction. Therefore, $W_{C P}(\phi)$ should be a monotone function in the angle range $0 \leq \phi \leq \pi / 4$ between the antinodal and nodal directions. However, the ARPES study $\underline{\underline{3}}$ shows unambiguously that the SC spectral weight turns out to be highly non-monotonic: 
at first, $W_{C P}(\phi)$ increases from zero at $\phi=\pi / 4$ up to a maximum at certain $\phi_{m}$ and then exhibits a considerable decrease if $\phi \rightarrow 0$. The spectral weight in the antinodal region is strongly dependent on doping. Such a non-monotonic behavior of $W_{C P}(\phi)$ is explained ${ }^{3}$ by a competition between superconductivity and an insulating state developing in the antinodal region with pronounced nesting feature of the FC. The insulating state should result in a depletion of the SC pairing channel and, in consequence of a decrease of the SC order parameter, in a lowering of the spectral weight of the SC coherent peak. It should be noted that the spectral weight in the antinodal region, observed by Kondo et al. $\frac{3}{\frac{3}{3}}$ is considerably greater than that corresponding to simple $\cos 2 \phi$ dependence as shown schematically in Fig. 5a.

The coherent peak disappears in the PG state above $T_{c}$ where spectral weight $W_{P G}(\phi)$ is zero in a broad angle range that can be referred to the nodal region (Fig. 5b). In the antinodal region, $W_{P G}(\phi)$ increases rapidly up to a maximum when $\phi \rightarrow 0$. Insulating order, which might be invoked to explain both $W_{C P}(\phi)$ and $W_{P G}(\phi)$, is not discovered for now. We believe that a competition of such a hidden order ${ }^{29}$ with superconductivity is not the only qualitative explanation of observed spectral properties in the antinodal region. We have shown that $\boldsymbol{K}$ - pairing concept $\mathrm{t}^{\frac{5}{}}$ leads to a consistent explanation of the origin of the SC and PG states: $\boldsymbol{K}$ - pairing in the antinodal region gives rise to both these states. A decrease of $W_{C P}(\phi)$ at $\phi \rightarrow 0$ can be associated with non-trivial zero lines of the SC order parameter $\Delta_{s c}(\boldsymbol{k})$ shown in Fig. 3 that is can be explained in just the same way as a decrease of $W_{C P}(\phi)$ at $\phi \rightarrow \pi / 4$ due to $d$-wave node. It is evident that such angle dependence of the spectral weight of the coherent peak, appearing in both nodal and antinodal regions owing to different microscopic mechanisms of SC pairing, can be considered as a direct indication of the biordered SC state. On the contrary, since the PG state is associated with QSS wave function, one can expect that $W_{P G}(\phi)=0$ in the nodal region whereas nonzero $W_{P G}(\phi)$ in the antinodal one is compared with $W_{C P}(\phi)$ that appears there below $T_{c}$. Due to a random phase of the wave function of QSS and corresponding gap function $\Delta_{p g}(\boldsymbol{k})$, nodal lines of these functions, that could be apparent in the antinodal region, cannot give a detectable contribution into a decrease of the spectral weight at $\phi \rightarrow 0$. Expected angle dependencies of $W_{C P}(\phi)$ and $W_{P G}(\phi)$ are shown schematically in Fig. 5.

We believe the antinodal region with pronounced nesting of the FC gives rise to $\boldsymbol{K}$-pairing whereas conventional pairing with $\boldsymbol{K}=0$ develops in the nodal region where the FC shows no signs of nesting. Thus, we do not oppose $\boldsymbol{K}$-pairing with the conventional pairing: these two SC pairing channels with slightly overlapped domains of definition in the momentum space form indivisible biordered SC state together. A passage from the antinodal region into the nodal one is accompanied with a redistribution of the spectral weight between these two pairing channels.

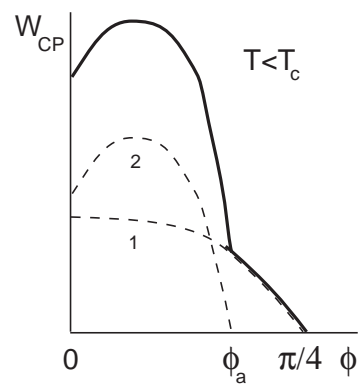

a

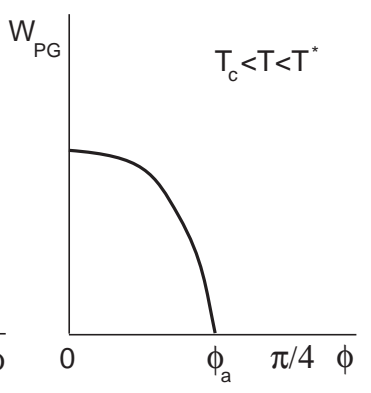

$\mathrm{b}$
FIG. 5: Angle dependence of the spectral weight (schematically). a. Spectral weight of the coherent SC peak (dashed lines 1 and 2 correspond to $d$-wave order parameter $(\sim \cos 2 \phi)$ and $\boldsymbol{K}$-pairing order parameter, respectively. b. Antinodal spectral weight of the PG state. Here, $\phi_{a}$ is a conditional angle boundary separating the nodal and antinodal regions.

\section{QUASIPARTICLE INTERFERENCE}

A rise of the coherence in the system of the antinodal $\boldsymbol{K}$-pairs below $T_{c}$ should inevitably lead to interference effects inherent in the SC state. Bogoliubov QPI appears due to mixing of quasipatricle states with high spectral weight that results in a modulation of the local density of states (LDOS) in the real space. Such states, at given quasiparticle energy (4), are disposed in vicinities of the points corresponding to maximal curvature of the isoline $E\left(k_{x}, k_{y}\right)=E=$ const. In the case of biordered SC state, pairing with zero total momentum dominates $\boldsymbol{K}$-pairing in the nodal region, therefore, $\eta(\boldsymbol{k}) \equiv 0$ in this region due to the fact that $\varepsilon(-\boldsymbol{k})=\varepsilon(\boldsymbol{k})$. Thus, quasiparticle spectrum in the nodal region turns out to be fully symmetrical with respect to the Fermi level.

On the contrary, $\boldsymbol{K}$-pairing dominates the pairing with zero momentum in the antinodal region including near rectilinear segments of the isolines in relatively small vicinity of the $\mathrm{FC}$ which primarily forms the singularity in the self-consistency equation. Because $\varepsilon(\boldsymbol{K} / 2+\boldsymbol{k}) \approx$ $\varepsilon(\boldsymbol{K} / 2-\boldsymbol{k})$ in this vicinity, quasiparticle spectrum in the antinodal region should be slightly asymmetrical with respect to the Fermi level. Due to the fact that there is a considerable increase of the deviation from mirror nesting in a vicinity of conditional boundary separating nodal and antinodal regions, one can expect a pronounced increase of the asymmetry of quasiparticle spectrum in this vicinity. Such a statement is compatible with photoemission data presented by Yang et al $\stackrel{30}{30}$ Also, it shows that an insulating state, competing with the SC one, cannot be considered as the only origin of the asymmetry ob-

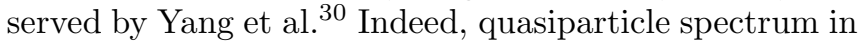
the case of insulating state with gap function $D(\boldsymbol{P})$ has the form

$$
E_{Q}(\boldsymbol{p})=\xi(\boldsymbol{p}) \pm \sqrt{\eta^{2}(\boldsymbol{p})+D(\boldsymbol{p})^{2}},
$$

where $2 \xi(\boldsymbol{p}) \equiv \varepsilon(\boldsymbol{p})+\varepsilon(\boldsymbol{p}+\boldsymbol{Q}), 2 \eta(\boldsymbol{p}) \equiv \varepsilon(\boldsymbol{p})-\varepsilon(\boldsymbol{p}+\boldsymbol{Q})$. Therefore, imperfect nesting, that is a deviation from the 


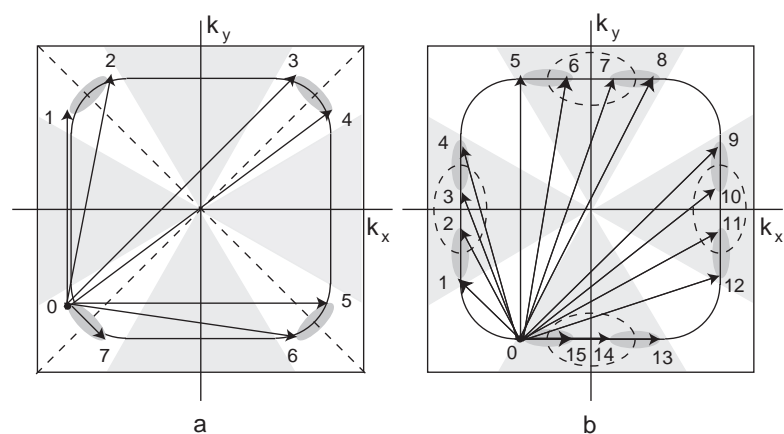

FIG. 6: Main scattering momenta determining nodal (a, in accordance with the "octet" model ${ }^{2}$ ) and antinodal (b) QPI pattern. FC and nodal line of the gap function are presented as solid and dashed lines, respectively. Small 'bananas' are showed as shadowed ovals. Antinodal sectors of the Brillouin zone are shadowed. Integers present the subscript enumerating main scattering momenta.

nesting condition (12), just as imperfect mirror nesting, results in the term $(\xi(\boldsymbol{p})$ or $\eta(\boldsymbol{p})$ before the square root) that originates electron-hole asymmetry in both cases.

To study antinodal quasiparticle spectrum qualitatively, one can neglect $\eta(\boldsymbol{k})$ in Eq. (16) owing to the fact that antinodal segments of the FC appear as near rectilinear. For this reason, at low quasiparticle energies, isoline shape can be analyzed in general form. In such a case, isolines enclose the gapless points of intersection of the FC and the nodal line. These singular points can be found from equation system $\xi_{K}\left(k_{x}, k_{y}\right)=0$, $\Delta_{K}\left(k_{x}, k_{y}\right)=0$.

The nodal part of the FC can be approximated by an arc of a circle whereas the nodal lines of $d$ - wave superconductor are straight lines $k_{y}= \pm k_{x}$. As a result, the quasiparticle isolines become apparent as 'banana'like closed curves 131 Because Fermi energy $\varepsilon_{F}$ exceeds $d$ - wave SC gap magnitude $\Delta_{m}$ considerably, $\varepsilon_{F} \gg \Delta_{m}$, 'banana' turns out to be very thin so that exactly its end points correspond to maximal curvature of the isoline. This directly leads to the octet model of QPI in the nodal region,,$\underline{\underline{31}}$ which defines a set of wave-vectors $\boldsymbol{k}_{i}$ $(1 \leq i \leq 8)$, corresponding to such end points, that determine LDOS pattern at given quasiparticle energy $E$. The octet model is presented in Fig. 5a where we define the main scattering momenta as $\boldsymbol{q}_{i}=\boldsymbol{k}_{i}-\boldsymbol{k}_{1}$. It should be noted that our definition of $\boldsymbol{q}_{i}$ is somewhat different from that given by Kohsaka et el $\stackrel{\underline{2}}{\underline{2}}$ These two definitions are mutually complementary in the reciprocal lattice.

As one can see from Fig. 3, singular points of antinodal quasiparticle spectrum $E(\boldsymbol{k})$ are symmetrically, with respect to the antinodal directions, disposed on near nested pieces of the FC. To study a shape of the isolines, one can approximate these pieces by straight lines and any of the nodal lines in a small vicinity of the singular point by an arc of a circle. Evidently, under the condition that $\varepsilon_{F} \gg \Delta_{m}$, all isolines in this vicinity are 'bananas' enveloping a rectilinear part of the FC. Indeed, if $E \ll \varepsilon_{F}$, an isoline of the the quasiparticle dispersion can be written in the form

$$
\kappa_{x}^{2}-\kappa_{0}^{2}=-\kappa_{y}^{2} \pm \alpha \sqrt{\kappa_{E}^{2}-\kappa_{y}^{2}}
$$

where $\kappa_{x}=k_{x} / k_{F}, \kappa_{y}=k_{y} / k_{F}$ are dimensionless components of the relative motion momentum, $k_{F}$ is the Fermi momentum in the antinodal direction, $\kappa_{0}$ is dimensionless radius of the nodal line, $\kappa_{E}=E / 2 \varepsilon_{F}$, $\alpha=2 \varepsilon_{F} / \Delta_{m}\left(k_{F} a\right)^{2}$. As follows from Eq. (17), there are closed isolines only under condition that $-\kappa_{E} \leq$ $\kappa_{y} \leq \kappa_{E}$. Therefore, a transversal, with respect to the $\mathrm{FC}$, size of the isoline equals $\kappa_{t}=2 \kappa_{E} \ll 1$. If $E \rightarrow 0$, closed isolines shrink into two singular points $\left( \pm \kappa_{0}, 0\right)$. A longitudinal size can be estimated as $\kappa_{l}=$ $\sqrt{\kappa_{0}^{2}+\alpha \kappa_{E}}-\sqrt{\kappa_{0}^{2}-\alpha \kappa_{E}}$, therefore, closed isolines appear in quasiparticle energy range $0<E \leq \Delta_{m}\left(k_{0} a\right)^{2}$. In addition, one can examine that $\kappa_{t} \ll \kappa_{l}$. Energy $E_{m}=\Delta_{m}\left(k_{0} a\right)^{2}$ corresponds to a topological transition from closed, at $E<E_{m}$, to opened, at $E>E_{m}$, isolines. Because opened isoline has no points of considerable curvature, the topological transition should result in a degradation of the interference pattern. Contrariwise, due to a large curvature of the closed isoline in small vicinities of its end points, exactly these vicinities should primarily contribute into the QPI. Therefore, following McElroy et al. $\stackrel{31}{,}$ one can introduce a set of momenta $\boldsymbol{q}_{i}(E)$ connecting different end points. Here, subscript $i$ runs from 1 to $2 n-1$ where $n$ is a number of singular points of quasiparticle dispersion (16). Such main scattering momenta, defined as $\boldsymbol{q}_{i}=\boldsymbol{k}_{i}-\boldsymbol{k}_{1}$ for any $i \neq 1$, should determine the real-space interference pattern. The pattern caused by the antinodal QPI turns out to be considerably more complicated in comparison with the nodal one even in the simplest case corresponding to the only closed nodal line in each of four crystal equivalent parts of the antinodal region as shown in Fig. 6b. The full set of momenta $\boldsymbol{q}_{i}$, following from non-trivial momentum dependence of the gap function shown in Fig. 3, should result in the real-space antinodal QPI pattern that can be considered as originating from fairly uniform distribution of scattering momenta. Therefore, it seems highly probable that the antinodal QPI pattern should be considerably more smooth with respect to the nodal one.

It is clear that due to expansion of the closed isolines $E(\boldsymbol{k})=E$ with an increase of $E$, there is a variation (rotation and decrease or increase of the absolute value) of the main scattering momenta, $\boldsymbol{q}_{i}=\boldsymbol{q}_{i}(E)$ with $E$. All of the nodal scattering momenta are dispersive, varying with $E$ in accordance with the octet model $\stackrel{2}{=}$ On the contrary, among the antinodal scattering momenta, there are some non-dispersive, such as "immobile" $\boldsymbol{q}_{5}$ and $\boldsymbol{q}_{14}$ shown in Fig. 6b, that, owing to nesting feature of the FC, remain independent of $E$ at small quasiparticle energies. Strictly speaking, only such "immobile" $\boldsymbol{q}_{i}$ can contribute into checkerboard real-space modulation in the SC state $\underline{\underline{32}}$

Nodes of the antinodal quasiparticle spectrum result in the fact that, at a finite temperature, thermal equilibrium 
quasiparticles are excited not only near $d$ - wave nodes in the nodal region ${ }^{33}$ but in the antinodal one as well. Moreover, the equilibrium population of the antinodal quasiparticles may considerably exceed their population in vicinities of the $d$ - wave nodes. This may occur if line of zeroes of the antinodal gap function, in contrast to the nodal one, turns out to be close to the FC in its extended vicinity.

\section{CONCLUSION}

Our concept of $\boldsymbol{K}$-pairing in the cuprates is based on two complementary statements: $1^{\circ}$ screened Coulomb repulsion is the underlying SC pairing interaction; $2^{\circ}$ large momentum of $\boldsymbol{K}$-pair arises due to nesting feature of the FC. SC $\boldsymbol{K}$-pairing, prevailing in the antinodal region of the momentum space, leads directly to uniform explana- tion of spatial inhomogeneity of both SC state in the form of checkerboard PDW and striped PG state formed by incoherent $\boldsymbol{K}$-pairs. $\boldsymbol{K}$-pairing, together with the conventional SC pairing with zero momentum prevailing in the nodal region, results in an indivisible biordered SC state which naturally explains the peculiarities of the angle dependence of the spectral weight both below and above $T_{c}$. Complicated momentum dependence of the gap function in the antinodal region should lead to fairly reach antinodal QPI resulting in relatively smooth realspace interference pattern.

\section{Acknowledgments}

This work was supported in part by the Russian Foundation for Basic Research (Project Nos. 08-02-00490 and 09-02-00682).
1 M.R. Norman, H. Ding, M. Randeria, J.C. Campuzano, T. Yokoya, T. Takeuchi, T. Takahashi, T. Mochiku, K. Kadowaki, P. Guptasarma, and D.G. Hink, Nature 392, 157 (1998).

2 Y. Kohsaka, C. Taylor, P. Wahl, A. Schmidt, Jhinhwan Lee, K. Fujita, J.M. Alldredge, K. McElroy, Jinho Lee, H. Eisaki, S. Uchida, D.H. Lee, and J.C. Davis, Nature 454, 1072 (2007).

3 T. Kondo, R. Khasanov, T. Takeuchi, J. Schmalian, and A. Kaminski, Nature 457, 296 (2009).

4 G. Deutscher, Nature 397, 410 (1999).

5 V.I. Belyavsky and Yu.V. Kopaev, Phys. Rev. B 76, 214506 (2007)

6 V.I. Belyavsky, Yu.V. Kopaev, V.M. Sofronov, and S.V. Shevtsov, JETP 97, 1032 (2003).

7 V.I. Belyavsky, Yu.V. Kopaev, Yu.N. Togushova, and S.V. Shevtsov, JETP 99, 585 (2004).

8 G.A. Gamov, Zs. f. Phys. 51, 204 (1928).

9 V.J. Emery and S.A. Kivelson, Nature 374, 434 (1995).

10 A.A. Abrikosov, L.P. Gorkov, and I.E. Dzyaloshinskii, Methods of Quantum Field Theory in Statistical Physics, revised ed., edited by R.A. Silverman (Dover, New York, 1975).

11 V.I. Belyavsky, V.V. Kapaev, and Yu.V. Kopaev, JETP Letters 86, 404 (2007).

12 A. Leggett, Nature Physics 2, 134 (2006).

13 D.N. Basov and T. Timusk, Rev. Mod. Phys. 77, 721 (2005).

14 A.M. Tsvelik and A.V. Chubukov, Phys. Rev. Lett. 98, 237001 (2007).

15 H.-D. Chen, O. Vafek, A. Yazdani, and S.-C. Zhang, Phys. Rev. Lett. 93, 187002 (2004).

16 J. Bardeen, L.N. Cooper, and J.R. Schrieffer, Phys. Rev. 1081175 (1957).

17 N.N. Bogoliubov, V.V. Tolmachev, and D.V. Shirkov, A New Method in the Theory of Superconductivity (Consultants Bureau, New York, 1959).
18 P. Fulde and A.R. Ferrell, Phys. Rev. 135, A550 (1964).

19 A.I. Larkin and Yu.N. Ovchinnikov, Sov. Phys. JETP 20, 762 (1965).

20 A. Ino, C. Kim, M. Nakamura, T. Yoshida, T. Mizokawa, A. Fujimori, Z.-X. Shen, T. Kakeshita, H. Eisaki, and S. Uchida, Phys. Rev. B 65, 094504 (2002).

21 W.D. Wise, M.C. Boyer, Kamalesh Chatterjee, Takeshi Kondo, T. Takeuchi, H. Ikuta, Yayu Wang, and E.W. Hudson Nature Physics 4, 696 (2008).

22 V.I. Belyavsky, Yu.V. Kopaev, N.T. Nguyen, and V.L. Tran, JETP 135, 340 (2009).

${ }^{23}$ K.M. Shen, F. Ronning, D.H. Lu, F. Baumberger, N.J.C. Ingle, W.S. Lee, W. Meevasana, Y. Kohsaka, M. Azuma, M. Takano, H. Takagi, and Z.-X. Shen, Science 307, 901 (2005).

24 A.I. Rusinov, Do Chan Kat and Yu.V. Kopaev, Sov. Phys. JETP 38, 991 (1974).

25 N. Furukawa, T.M. Rice, and M. Salmhofer, Phys. Rev. Lett. 81, 3195 (1998).

26 W. Kohn and J.M. Luttinger, Phys. Rev. Lett. 15, 24 (1965).

27 E. Berg, E. Fradkin, and S.A. Kivelson, Phys. Rev. B 79, 064515 (2009).

28 W.-F. Tsai, H. Yao, A. Läuchli, and S.A. Kivelson, Phys. Rev. B 77, 214502 (2008).

29 S. Chakravarty, R.B. Laughlin, D.K. Morr, and C. Nayak, Phys. Rev. B 63, 094503 (2001).

${ }^{30}$ H.-B. Yang, J.D. Rameau, P.D. Johnson, T. Valla, A. Tsvelik, and G.D. Gu, Nature 456, 77 (2008).

31 K. McElroy, R.W. Simmonds, J.E. Hoffman1, D.-H. Lee, J. Orenstein, H. Eisaki, S. Uchida, and J.C. Davis, Nature 422, 592 (2003).

32 Q-H. Wang and D-H. Lee, Phys. Rev. B 67, 020511(R) (2003).

33 N. Gedik, J. Orenstein, Ruixing Liang, D.A. Bonn, and W.N. Hardy, Science 300, 1410 (2003). 